\title{
ON NON-CROSS VARIETIES OF GROUPS
}

\author{
L. G. KOVÁCS and M. F. NEWMAN
}

(Received 25 July 1969)

To Bernhard Hermann Neumann on his 60th birthday

(Communicated by G. E. Wall)

\section{Introduction}

Our title has become something of a misnomer, however we retain it since drafts of this note have been quoted with it.

Unless otherwise stated our terminology and notation follow that in Hanna Neumann's book [12].

The Oates-Powell Theorem ([12] p. 151) allows us to say that a variety is Cross if and only if it can be generated by a finite group, and to assert that the laws of a Cross variety are finitely based. A variety is just-non-Cross if it is not Cross but every proper subvariety of it is Cross.

We asked in [9]: what non-Cross varieties have just-non-Cross subvarieties? The answer is: all of them.

THEOREM 1. Every non-Cross variety has a just-non-Cross subvariety.

The proof is an easy application of Zorn's Lemma. If $\left\{\mathfrak{B}_{\lambda}: \lambda \in \Lambda\right\}$ is a descending chain of non-Cross subvarieties of a non-Cross variety such that the intersection $\mathfrak{D}=\wedge\left\{\mathfrak{B}_{\lambda}: \lambda \in \Lambda\right\}$ is properly contained in each $\mathfrak{B}_{\lambda}$, then the union of the corresponding chain $\left\{B_{\lambda}: \lambda \in A\right\}$ of fully invariant subgroups of the word group $X_{\infty}$ ([12] p. 4) is not finitely generated, hence $\mathfrak{D}$ is not finitely based, and a fortiori $D$ is still non-Cross.

In [9] we claimed that for every prime $p$ the product variety $\mathfrak{A}_{p} \mathfrak{A}_{p}$ is just-nonCross $\left(\mathfrak{H}_{p}\right.$ is the variety of abelian groups of exponent dividing $\left.p\right)$. Here we substantiate this as a consequence of a detailed description, in section 2 , of the lattice of subvarieties of $\mathfrak{A}_{p^{\alpha}} \mathfrak{A}_{p}$.

The variety $\mathfrak{A}$ of all abelian groups and the varieties $\mathfrak{H}_{p} \mathfrak{A}_{p}$ are just-non-Cross and nilpotent-by-abelian. The converse is also true.

THEOREM 2. The only nilpotent-by-abelian just-non-Cross varieties are $\mathfrak{A}$ and the $\mathfrak{A}_{p} \mathfrak{A}_{p}$.

This theorem is related to the so-called external result we state in section 3, and is proved with it in section 5. 


\section{The subvariety lattice of $\mathfrak{A}_{p^{\alpha}} \mathfrak{A}_{p}$}

In this section we give a description of the lattice of subvarieties of $\mathfrak{A}_{p^{x}} \mathfrak{A}_{p}$. Proofs are deferred to section 4.

Lattice terminology follows Birkhoff [1].

We begin with some notation. The set of positive integers is denoted by $P$. As usual $\mathfrak{A}_{n}, \mathfrak{B}_{n}, \mathfrak{R}_{n}$ denote, respectively, the variety of abelian groups of exponent dividing $n$, the variety of groups of exponent dividing $n$, and the variety of groups of nilpotency class at most $n$. The variety of all groups will, for convenience, be denoted $\mathfrak{R}_{\omega}$. Our description of the subvarieties of $\mathfrak{A}_{p^{\alpha}} \mathfrak{A}_{p}$ will be in terms of these varieties and one more family whose members will be denoted $\mathfrak{A}_{n *}$. The variety $\mathfrak{N}_{n *}$ is the subvariety of $\mathfrak{R}_{n}$ defined by the additional law $\prod_{s=2}^{n}\left[x_{s}, x_{1}, \cdots, x_{s-1}\right.$, $\left.x_{s+1}, \cdots, x_{n}\right]$. Note that $\mathfrak{N}_{n *} \supseteq \mathfrak{N}_{n-1}$. For any particular prime $p$ only certain of these additional varieties are needed, namely those for which $n$ is at least 3 and is divisible by $p$. We therefore introduce for each prime $p$ an ordered extension $P(p)$ of $P$ defined by:

$$
\begin{aligned}
& P(p)=\{1, \cdots, p-1, p *, p, \cdots, p r-1, p r *, p r, \cdots, \omega\} \quad \text { for } p \text { odd } \\
& P(2)=\{1,2,3,4 *, 4, \cdots, 2 r-1,2 r * 2 r, \cdots, \omega\}
\end{aligned}
$$

with the order as indicated. The $P(p)$ and $\{0,1, \cdots, \alpha+1\}$ taken in this order may be considered as lattices - we do this. For each $p$ the varieties $\mathfrak{B}_{p^{\beta}}$ and $\mathfrak{A}_{p^{z}} \mathfrak{A}_{v}$ for $v$ in $P(p)$ play a distinguished role. We denote them $\mathfrak{B}(\beta)$ and $\mathfrak{N}(\tau, v)$ respectively.

With each subvariety $\mathfrak{B}$ of $\mathfrak{A}_{p^{2}} \mathfrak{A}_{p}$ we associate an element $\boldsymbol{\beta}(\mathfrak{B})$ of $\{0, \cdots$, $\alpha+1\}$ and elements $v(0, \mathfrak{B}), \cdots, v(\alpha-1, \mathfrak{B})$ of $P(p)$ as follows:

for $\tau \in\{0, \cdots, \alpha-1\}$,

$$
\boldsymbol{\beta}(\mathfrak{B})=\min \{\beta: \mathfrak{B} \subseteq \mathfrak{B}(\beta)\} ;
$$

$$
\boldsymbol{v}(\tau, \mathfrak{B})=\min \{v: \mathfrak{B} \subseteq \mathfrak{N}(\tau, v)\} .
$$

The subvarieties of $\mathfrak{A}_{p^{*}} \mathfrak{A}_{p}$ are characterized by the above invariants:

2.1 If $\mathfrak{B}$ is a subvariety of $\mathfrak{A}_{p^{\alpha}} \mathfrak{A}_{p}$, then

$$
\mathfrak{B}=\mathfrak{A}_{p^{\alpha}} \mathfrak{A}_{p} \wedge \mathfrak{B}(\boldsymbol{\beta}(\mathfrak{B})) \wedge \bigwedge_{\tau=0}^{\alpha-1} \mathfrak{N}(\tau, v(\tau, \mathfrak{B})) .
$$

If $\alpha=1$, it follows that every proper subvariety of $\mathfrak{H}_{p} \mathfrak{H}_{p}$ is nilpotent, and hence Cross because it has finite exponent. As $\mathfrak{A}_{p} \mathfrak{A}_{p}$ is obviously not Cross, this yields the following.

THEOREM 3. For every prime $p$ the variety $\mathfrak{A}_{p} \mathfrak{H}_{p}$ is just-non-Cross.

This discharges a debt incurred in [9]. The proof here - due primarily to one of us (MFN) - supersedes an earlier one which motivated the papers [4], [5] (and in which the result was also announced).

It is clear that for all subvarieties $\mathfrak{U}, \mathfrak{B}$ of $\mathfrak{A}_{p^{\alpha}} \mathfrak{A}_{p}$ 


$$
\boldsymbol{\beta}(\mathfrak{U} \vee \mathfrak{B})=\max \{\boldsymbol{\beta}(\mathfrak{U}), \boldsymbol{\beta}(\mathfrak{V})\}
$$

and

$$
\boldsymbol{v}(\tau, \mathfrak{U} \vee \mathfrak{B})=\max \{\boldsymbol{v}(\tau, \mathfrak{U}), \boldsymbol{v}(\tau, \mathfrak{B})\}
$$

for all $\tau$ in $\{0, \cdots, \alpha-1\}$. The next point to prove is that the corresponding result for meets also holds.

2.2 For all subvarieties $\mathfrak{U}, \mathfrak{B}$ of $\mathfrak{A}_{p^{z}} \mathfrak{A}_{p}$

and

$$
\boldsymbol{\beta}(\mathfrak{U} \wedge \mathfrak{B})=\min \{\boldsymbol{\beta}(\mathfrak{U}), \boldsymbol{\beta}(\mathfrak{B})\}
$$

$$
\boldsymbol{v}(\tau, \mathfrak{U} \wedge \mathfrak{B})=\min \{v(\tau, \mathfrak{U}), \boldsymbol{v}(\tau, \mathfrak{B})\}
$$

for all $\tau$ in $\{0, \cdots, x-1\}$.

Now it follows from 2.1 that the mapping $\chi: \mathfrak{B} \mapsto(\beta(\mathfrak{B}), \boldsymbol{v}(0, \mathfrak{B}), \cdots$, $\boldsymbol{v}(\alpha-1, \mathfrak{B}))$ is an embedding of the lattice of subvarieties of $\mathfrak{A}_{p^{\alpha}} \mathfrak{A}_{p}$ into the direct product $A$ of the lattice $\{0, \cdots, \alpha+1\}$ with $\alpha$ copies of $P(p)$. A sublattice of a direct product of distributive lattices with descending chain condition is a distributive lattice with descending chain condition.

THEOREM 4. The lattice of subvarieties of $\mathfrak{U}_{p^{*}} \mathfrak{H}_{p}$ is distributive with descending chain condition.

The description of the lattice of subvarieties of $\mathfrak{A}_{p^{\alpha}} \mathfrak{A}_{p}$ is now completed by giving its image under $\chi$. Let $\Sigma$ be the subset of the direct product lattice $\Lambda$ defined by:

$$
\begin{aligned}
& \left(\beta, v_{0}, \cdots, v_{x-1}\right) \in \Sigma \text { if and only if } \\
& v_{\beta}=\cdots=v_{\alpha-1}=1 \text { for } \beta<\alpha, \\
& v_{\beta-1}<p \quad \text { for } 1 \leqq \beta \leqq \alpha \text {; } \\
& \begin{cases}v_{\tau} & \text { for } v_{\tau} \in\{1, \omega\}, \\
v_{\tau}-p+1 & \text { for } v_{\tau} \in P \text { and }\end{cases} \\
& v_{\tau+1} \leqq \begin{cases}v_{\tau}-p+1 & \text { for } v_{\tau} \in P \text { and } v_{\tau}>p, \\
p r & \text { for } v_{\tau}=p(r+1) * \text { with } r \in P, \\
2 & \text { for } 2 \leq v_{\tau} \leq p ;\end{cases} \\
& \text { for } 2 \leqq v_{\tau} \leqq p \text {; } \\
& v_{\tau+2}=1 \quad \text { for } v_{\tau} \leqq 2 p-1 .
\end{aligned}
$$

2.3 The image of $\chi$ is $\Sigma$.

While the description of the lattice of subvarieties of $\mathfrak{U}_{p^{\alpha}} \mathfrak{A}_{p}$ afforded by all this is adequate, it is somewhat ad hoc. Because the lattice is distributive with descending chain condition, it follows (cf. section 2 of Chapter VIII of [1] suitably corrected) that every element of the lattice can be uniquely written as an irredundant finite join of (finitely) join-irreducible elements. Moreover, a finite set of join-irreducibles gives its join irredundantly if and only if no two distinct elements of the set are comparable. Hence such a lattice can easily be reconstructed from the partially ordered set of its join-irreducible elements. The reconstruction 
can be carried out so as to yield a faithful representation of the lattice in the lattice of all subsets of the set of its join-irreducible elements. These facts suggest that a canonical way of describing such lattices is to give the partially ordered sets of their join-irreducible elements. We do this for the lattice of subvarieties of $\mathfrak{A}_{p^{x}} \mathfrak{A}_{p}$. An advantage of this approach is that our results are then more readily comparable with related results of Brooks [2] and Bryce [3], and better suited for the extension of the present results to a description of the subvarieties of $\mathfrak{A H}_{n}$ with square-free $n$ (to be given in [10]).

Given 2.3 and the explicit description of the sublattice $\Sigma$ of $A$, it is an elementary exercise to derive the desired information. We simply give the result after a hint to the derivation we used.

If an element $\left(\beta, v_{0}, \cdots, v_{\alpha-1}\right)$ of $\Sigma$ is join-irreducible, then $\left(\beta^{\prime}, v_{0}, \cdots, v_{\alpha-1}\right)$ $\notin \Sigma$ for $\beta^{\prime}<\beta$ because

$$
\left(\beta, v_{0}, \cdots, v_{\alpha-1}\right)=(\beta, 1, \cdots, 1) \vee\left(\beta^{\prime}, v_{0}, \cdots, v_{\alpha-1}\right) .
$$

Similarly $\left(\beta, v_{0}, \cdots, v_{\tau-1}, \mu, v_{\tau+1}, \cdots, v_{\alpha-1}\right) \notin \Sigma$ for $\mu<v_{\tau}$ and $\tau \in\{0, \cdots, \alpha-2\}$. Hence if $v_{\alpha-1}=v \neq 1$, the conditions defining $\Sigma$ determine $\beta, v_{0}, \ldots, v_{\alpha-2}$;

$$
\begin{aligned}
& \beta= \begin{cases}\alpha & \text { for } v<p, \\
\alpha+1 & \text { for } v \geqq p ;\end{cases} \\
& v_{\tau}=\left\{\begin{array}{ll}
\omega & \text { for } v=\omega, \\
\langle v\rangle+(p-1)(\alpha-1-\tau) & \text { for } v \neq \omega
\end{array} \text { except } v=2, \tau \in\{\alpha-3, \alpha-2\} ;\right. \\
& v_{x-2}=2, v_{\alpha-3}=2 p * \quad \text { for } v=2 \text { : }
\end{aligned}
$$

here, and in the sequel, $v \mapsto\langle v\rangle$ denotes the mapping of $P(p) \backslash\{\omega\}$ to $P$ which is the identity on $P$ and for which $\langle p r *\rangle=p r$ whenever $p r * \in P(p) \backslash P$. Finally, if $v_{\alpha-1}=1$, then $\beta=\alpha+1$ or the corresponding variety lies in $\mathfrak{A}_{p^{\alpha-1}} \mathfrak{A}_{p}$; if $\beta=\alpha+1$, then $v_{0}=\cdots=v_{\alpha-2}=1$. It is straightforward to check that the resulting elements of $\Sigma$ are join-irreducible.

We can now describe the partially ordered set $J\left(p^{\alpha}\right)$ of the join-irreducible subvarieties of $\mathfrak{A}_{p^{\alpha}} \mathfrak{H}_{p}$. Clearly $J\left(p^{0}\right)$ consists of $\mathbb{E}$ and $\mathfrak{A}_{p}$ with $\mathbb{E} \subset \mathfrak{A}_{p}$. For $\alpha$ in $P$ the set $J\left(p^{\alpha}\right)$ consists of $J\left(p^{\alpha-1}\right)$ and for each $v$ in $P(p)$ a variety $\mathfrak{s}\left(p^{\alpha}, v\right)$ defined as follows:

$$
\begin{aligned}
& \Im\left(p^{\alpha}, 1\right)=\mathfrak{A}_{p^{\alpha+1}} ; \\
& \Im\left(p^{\alpha}, 2\right)=\mathfrak{A}_{p^{\alpha}} \mathfrak{A}_{p} \wedge \mathfrak{B}_{p^{\alpha}} \wedge \mathfrak{R}_{2+(p-1)(\alpha-1)} \wedge \mathfrak{A}_{p^{\alpha-3}} \mathfrak{R}_{2 p^{*}} \wedge \mathfrak{U}_{p^{\alpha-2}} \mathfrak{N}_{2}
\end{aligned}
$$

here the second term must be omitted when $p=2$, and the fourth and fifth when they are not meaningful (also, the third term is redundant when $\alpha$ is 2 or 3 ); for $v \in P(p) \backslash\{1,2, \omega\}$,

$$
\mathfrak{J}\left(p^{\alpha}, v\right)=\mathfrak{A}_{p^{\alpha}} \mathfrak{U}_{p} \wedge \mathfrak{B}_{p^{\alpha}} \wedge \mathfrak{R}_{\langle v\rangle+(p-1)(\alpha-1)} \wedge \mathfrak{U}_{p^{\alpha-1}} \mathfrak{N}_{v}
$$

here the second term must be omitted when $v \geqq p$ and the last term is redundant when $v \in P$; and 


$$
\mathfrak{\Im}\left(p^{\alpha}, \omega\right)=\mathfrak{A}_{p^{\alpha}} \mathfrak{A}_{p} .
$$

Note that the only non-nilpotent join-irreducible varieties in $\mathfrak{A}_{p^{\alpha}} \mathfrak{A}_{p}$ are the $\mathfrak{A}_{p^{\tau}} \mathfrak{A}_{p}$ with $\tau \in\{1, \cdots, \alpha\}$. In contrast to this Brooks [2] has shown that there is an infinite number of non-nilpotent join-irreducible subvarieties in $\mathfrak{A}_{p} \mathfrak{A}_{p^{2}}$.

It is a routine matter to check that the partial order on $J\left(p^{\alpha}\right)$ is generated by that on $J\left(p^{x-1}\right)$ and the inclusions:

$$
\begin{aligned}
& \mathfrak{H}_{p} \subset \mathfrak{\Im}\left(p^{\alpha}, 1\right) \subset \mathfrak{\Im}\left(p^{\alpha}, p\right), \\
& \mathfrak{A}_{p} \subset \mathfrak{I}\left(p^{\alpha}, 2\right), \\
& \mathfrak{S}\left(p^{\alpha}, \mu\right) \subset \mathfrak{\Im}\left(p^{\alpha}, v\right) \quad \text { whenever } \mu, v \in P(p) \text { and } 2 \leqq \mu<v ;
\end{aligned}
$$

if $\alpha>1$ then also

$$
\begin{aligned}
& \Im\left(p^{\alpha-1}, 1\right) \subset \Im\left(p^{\alpha}, 1\right), \\
& \Im\left(p^{\alpha-1}, 1\right) \subset \mathfrak{\Im}\left(p^{\alpha}, 2\right), \\
& \Im\left(p^{\alpha-1}, 2\right) \subset \mathfrak{\Im}\left(p^{\alpha}, 2\right), \\
& \Im\left(p^{\alpha-1},\langle v\rangle+p-1\right) \subset \Im\left(p^{\alpha}, v\right) \text { for all } v \text { in } P(p) \backslash\{1,2, \omega\}, \\
& \Im\left(p^{\alpha-1}, \omega\right) \subset \mathfrak{\Im}\left(p^{\alpha}, \omega\right) ;
\end{aligned}
$$

and if $\alpha>2$ then

$$
\mathfrak{\Im}\left(p^{\alpha-2}, 2 p *\right) \subset \mathfrak{\Im}\left(p^{\alpha}, 2\right) .
$$

It is easy to indicate diagrammatically the lattice in the case $\alpha=1$ and, say, $p \neq 2$ :

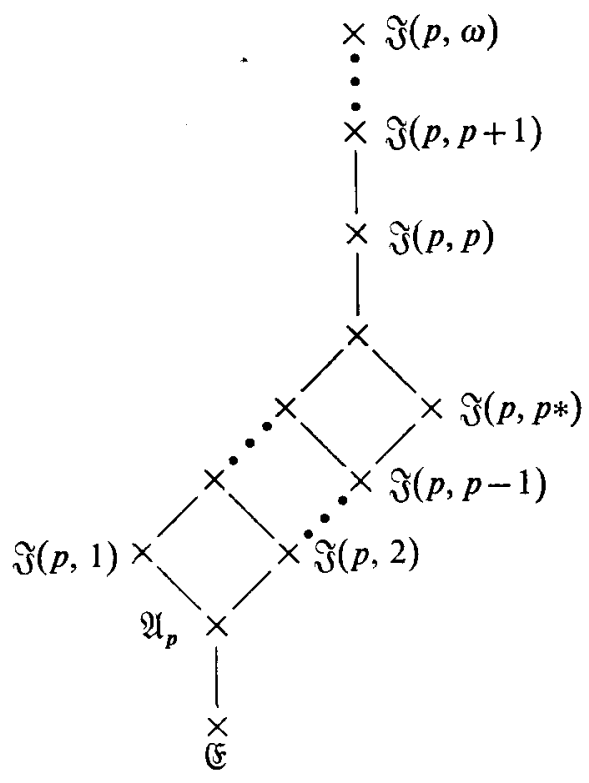




\section{External result on $\mathfrak{A}_{\boldsymbol{p}} \mathfrak{A}_{\boldsymbol{p}}$}

By an external result on a variety $\mathfrak{B}$ we mean a result of the form: A variety which does not contain $\mathfrak{B} \cdots$. For example, a variety which does not contain $\mathfrak{A}$ has finite exponent. For $\mathfrak{A}_{p} \mathfrak{A}_{p}$ we can prove the following.

THeORem 5. A soluble variety which does not contain $\mathfrak{2}_{p} \mathfrak{H}_{p}$ cannot contain any non-nilpotent p-group and therefore has a bound on the nilpotency class of its $p$ groups.

The proof is given in section 5 .

One might hope for a stronger result which we state as a problem.

In a variety which does not contain $\mathfrak{H}_{p} \mathfrak{H}_{p}$ is every locally finite p-group nilpotent?

The local finiteness is needed in view of the result of Novikov-Adyan [13] which implies that for all large enough primes $p$ there are infinite finitely generated groups of exponent $p$. Note also that this result implies the existence of just-nonCross varieties of exponent $p$.

A special case of the above problem is the well-known question: is there a bound on the nilpotency class of finite groups of exponent $p$ ?

It is perhaps worth recording some consequences of Theorem 5.

Corollary 1. A soluble variety which does not contain $\mathfrak{A}_{p} \mathfrak{A}_{p}$ has a bound on the nilpotency class of nilpotent torsion free groups in it.

COROLlary 2. A soluble variety in which the nilpotent groups do not form a subvariety contains $\mathfrak{A}_{p} \mathfrak{A}_{p}$ for some prime $p$.

This discharges another debt incurred in [9].

COROLlaRY 3. The variety generated by the two-generator free metabelian-ofexponent- $q$ groups for an infinite set of primes $q$ contains $\mathfrak{A}_{p} \mathfrak{H}_{p}$ for some prime $p$.

The last statement is in fact valid for all $p$ but this requires additional argument which is not given in this note.

\section{Proofs for section 2}

Most of the discussion is set in a free group $H$ of $\mathfrak{A}_{p^{\alpha}} \mathfrak{A}_{p}$ of countably infinite rank freely generated by $\left\{a_{i}: i \in P\right\}$. Much of the argument will involve the verbal subgroups $\mathfrak{H}_{p}(H), \mathfrak{B}(\beta)(H)$ and $\mathfrak{N}(\tau, \nu)(H)$; we denote them $A_{p}, B(\beta), N(\tau, v)$ respectively.

We first write down relationships between the subgroups $N(\tau, v)$. The first two are obvious:

4.01 For all $\tau$ in $\{0\} \cup P$ and all $\mu \leqq v$ in $P(p)$,

$$
N(\tau, \mu) \geqq N(\tau, v) \text { and } \mathfrak{U}_{p}(N(\tau, v))=N(\tau+1, v) \text {. }
$$

Further relations are easy consequences of some well-known results about 
commutators. We record here all such results which are used frequently in what follows. Notation: $[u, v]=u^{-1} v^{-1} u v,[u, v, w]=[[u, v], w],[u, 0 v]=u$ and $[u, n v]=[u,(n-1) v, v]$ for all $n$ in $P$. The identity is denoted $e$.

4.02 For $h, h_{1}, h_{2}, \cdots$ in $H$ and $d$ in $A_{p}$,

$$
\begin{aligned}
{\left[h_{1}, h_{2} h_{3}\right] } & =\left[h_{1}, h_{2}\right]\left[h_{1}, h_{3}\right]\left[h_{1}, h_{2}, h_{3}\right] ; \\
{\left[h_{1} h_{2}, h_{3}\right] } & =\left[h_{1}, h_{3}\right]\left[h_{2}, h_{3}\right]\left[h_{1}, h_{3}, h_{2}\right] ; \\
{\left[h_{1}, h_{2}, h_{3}\right] } & =\left[h_{1}, h_{3}, h_{2}\right]\left[h_{3}, h_{2}, h_{1}\right] ; \\
{\left[d, h_{1}, \cdots, h_{m}\right] } & =\left[d, h_{1 \pi}, \cdots, h_{m \pi}\right]
\end{aligned}
$$

for all $m$ in $P$ and all permutations $\pi$ of $\{1, \cdots, m\}$;

$$
\left[h_{1}, h_{2}^{m}\right]=\prod_{i=1}^{m}\left[h_{1}, i h_{2}\right]^{m ! / i !(m-i) !} \quad \text { for all } m \text { in } P
$$

in particular, since $\left[d, h^{p}\right]=e$ and $\left[h_{1}^{p}, h_{2}^{p}\right]=e$,

and

$$
\prod_{i=1}^{p}[d, i h]^{p ! / i !(p-i) !}=e
$$

$$
\prod_{i=1}^{p} \prod_{j=1}^{p}\left[h_{2}, i h_{1},(j-1) h_{2}\right]^{(p !)^{2} / i ! j !(p-i) !(p-j) !}=e .
$$

The last two equations have the following immediate consequences.

4.03 For all $\tau$ and all $n$ in $P$,

$$
N(\tau+1, n+1) \leqq N(\tau, n+p) \text { and } N(\tau+2,1) \leqq N(\tau, 2 p-1) .
$$

In fact a little more is true.

4.04 For all $\tau$ and all $r$ in $P$,

$$
N(\tau+1, p r) \leqq N(\tau, p(r+1) *) .
$$

Before proving this, we introduce some further notation. For $s, n$ in $P$ with $2 \leqq s<n$, let

and

$$
b(s, s)=\left[a_{s}, a_{1}, a_{2}, \cdots, a_{s-1}\right]
$$

$$
b(s, n)=\left[b(s, n-1), a_{n}\right] .
$$

We denote by $\imath$ the identity endomorphism of $H$, and by $\pi_{i, j}$ with $i, j$ in $P$ the endomorphism which fixes all the generators except $a_{i}, a_{j}$ which it interchanges.

ProOF OF 4.04. Let $\psi$ be the endomorphism of $H$ which maps $a_{j}$ to $a_{p r+1}$ if $p r+1 \leqq j \leqq p(r+1)$ and to $a_{j}$ otherwise. From

$$
\prod_{s=2}^{p(r+1)}(b(s, p(r+1)) \psi)^{p^{\tau}} \in N(\tau, p(r+1) *)
$$


it is easy to derive, using 4.02 and the inclusions

$$
N(\tau+1, p r+1) \leqq N(\tau, p(r+1)) \leqq N(\tau, p(r+1) *)
$$

(which hold on account of 4.03 and 4.01 ), that

$$
h=\prod_{s=2}^{p r} b(s, p r+1)^{p^{\tau+1}} \in N(\tau, p(r+1) *) .
$$

Then, applying $l-\pi_{2, p r+1}$ to $h$ and using 4.02 , one gets

$$
\left[a_{2}, a_{p r+1}, a_{1}, a_{3}, a_{4}, \cdots, a_{p r}\right]^{p^{+1}} \in N(\tau, p(r+1) *)
$$

and the result follows.

The story is completed by obtaining suitable generating sets for the $N(\tau, v)$.

Let $\mathscr{B}$ be the subset of $H$ defined by: $b \in \mathscr{B}$ if and only if $b=\left[a_{i}, m_{j} a_{j}\right.$, $\left.m_{j+1} a_{j+1}, \cdots, m_{s} a_{s}\right]$ where $i>j \leqq s ; m_{j}-1, m_{j+1}, \cdots, m_{s} \in\{0, \cdots, p-1\}$, $m_{s} \neq 0$; and if $m_{j}=p$ then firstly $i \leqq s$ implies $m_{i}<p-1$ and secondly $m_{k}=0$ whenever $j<k<i$ and $k \leqq s$.

4.05 The set $\mathscr{B} \cup\left\{a_{i}^{p}: i \in P\right\}$ is a free generating set for $A_{p}$ as free $\mathfrak{A}_{p^{z}}$-group.

Proof. It follows easily from 4.02 that $A_{p}$ is generated by $\mathscr{B}^{*}=\mathscr{B} \cup\left\{a_{i}^{p}\right.$ : $i \in P\}$. If there were a non-trivial relation between the elements of $\mathscr{B} *$ this would involve only finitely many of $\left\{a_{i}: i \in P\right\}$. It therefore suffices to consider for each $k$ in $P$ the subgroup $H_{k}$ of $H$ generated by $\left\{a_{1}, \cdots, a_{k}\right\}$ and to show that $\mathfrak{A}_{p}\left(H_{k}\right) \cap \mathscr{B}^{*}$ is independent in $\mathfrak{A}_{p}\left(H_{k}\right)$. By the Schreier formula for the rank of subgroups of absolutely free groups, $\mathfrak{O}_{p}\left(H_{k}\right)$ has rank $(k-1) p^{k}+1$. On the other hand the number of elements in $\mathfrak{A}_{p}\left(H_{k}\right) \cap \mathscr{B}^{*}$ is

$$
k+\sum_{j=1}^{k-1}\left\{(k-j)(p-1) p^{k-j}+\sum_{i=j+1}^{k}(p-1) p^{k-i}\right\}
$$

where the first term in $\{\cdots\}$ comes from counting the commutators with $m_{j} \neq p$ and the second term from the rest. The sum comes to $(k-1) p^{k}+1$ and the result follows.

Note that this proof implies that every element of $\mathscr{B}$ can be uniquely written in the way it is defined.

It follows that the commutator subgroup $N(0,1)$ of $H$ is a free $\mathfrak{A}_{p^{x}}$-group freely generated by $\mathscr{B}$. The other terms $N(0, n)$ of the lower central series of $H$ are a little more complicated to describe. This we do next after first defining weights for elements of $\mathscr{B}$.

The weight wt $(b)$ of an element $b=\left[a_{i}, m_{j} a_{j}, \cdots, m_{s} a_{s}\right]$ of $\mathscr{B}$ is $1+\sum_{k=j}^{s} m_{k}$. The weight wt $\left(b, a_{k}\right)$ of $b$ in the generator $a_{k}$ is 'the number of occurrences of $a_{k}$ in $b$, that is, 


$$
\text { wt }\left(b, a_{k}\right)= \begin{cases}0 & \text { if } k \notin\{j, \cdots, s\} \cup\{i\}, \\ m_{k} & \text { if } k \in\{j, \cdots, s\} \backslash\{i\}, \\ m_{k}+1 & \text { if } k=i \in\{j, \cdots, s\}, \\ 1 & \text { if } k=i \notin\{j, \cdots, s\} .\end{cases}
$$

4.06 For $n$ in $P$ the subgroup $N(0, n)$ is generated by the set $\mathscr{B}_{n}$ of elements of the form $b^{p^{k}}$ where $b \in \mathscr{B}, k \in\{0, \cdots, \alpha-1\}$, wt $(b)+k(p-1)>n$ and $k \mathrm{wt}(b) \neq 2$ unless $n=1$.

Proof. A routine argument from 4.02 shows that $N(\tau, n)$ is generated by the $\left[a_{i_{1}}, \cdots, a_{i_{m}}\right]^{p^{k}}$ with $m>n, k \in\{\tau, \cdots, \alpha-1\}$ and $i_{1}>i_{2} \leqq i_{3} \leqq \cdots \leqq i_{m}$. From this and 4.03 one gets immediately that $N(0, n)$ is generated by the set $\mathscr{P}$ of the elements $\left[a_{i_{1}}, \cdots, a_{i_{m}}\right]^{p^{k}}$ with $m \geqq 2, k \in\{0, \cdots, \alpha-1\}, k m \neq 2$ unless $n=1$, $m+(p-1) k>n$ and $i_{1}>i_{2} \leqq \cdots \leqq i_{m}$. Clearly $\mathscr{B}_{n}$ is a subset of $\mathscr{S}$. An induction on $m$ using 4.02 shows that each element of $\mathscr{P}$ lies in the subgroup generated by $\mathscr{B}_{n}$. The result follows.

Note that if $b^{p^{k}} \in \mathscr{B}_{n}$ and $k<\alpha-1$, then $b^{p^{k+1}} \in \mathscr{B}_{n}$. Thus for all $n$ every element of $N(0, n)$ can be uniquely written (up to order) in the form $\prod_{i=1}^{t} b_{i}^{\beta(i)}$ where the $b_{i}$ are distinct elements of $\mathscr{B}_{n}$ and the $\beta(i) \in\{1, \cdots, p-1\}$.

Similar generating sets can be given for the $N(0, p r *)$.

4.07 For pr* in $P(p)$ the subgroup $N(0, p r *)$ is generated by the union $\mathscr{B}_{p r}$ of

(i) $\mathscr{B}_{p r}$,

(ii) the set of elements of the form $b^{p^{k+1}}$ where $b \in \mathscr{B}, k \in\{0, \cdots, \alpha-2\}$,

$\mathrm{wt}(b)+(k+1)(p-1)=p r$, and $k \mathrm{wt}(b) \neq 2$, and

(iii) the set of elements of the form $\prod_{s=2}^{p r} b(s, p r) \psi$ where $\psi$ is an endomorphism of $H$ such that $a_{j} \psi=a_{i_{j}}$ where $i_{1} \leqq i_{2} \leqq \cdots \leqq i_{p r}$ and no $p$ of $i_{2}, \cdots, i_{p r}$ are equal.

Proof. The argument is essentially the same as that in the proof of 4.06. It is routine to derive from 4.02 that $N(\tau, p r *)$ is generated by the $\left[a_{i_{1}}, \cdots a_{i_{m}}\right]^{p^{k}}$ with $m \geqq p r+1, k \in\{\tau, \cdots, \alpha-1\}$ and $i_{1}>i_{2} \leqq \cdots \leqq i_{m}$, and the

$$
\prod_{s=2}^{p r}\left[a_{i_{s}}, a_{i_{1}}, a_{i_{2}}, \cdots, a_{i_{s-1}}, a_{i_{s+1}}, \cdots, a_{i_{p r}}\right]^{p^{\tau}} \text { with } i_{1} \leqq i_{2} \leqq \cdots \leqq i_{p r} .
$$

Hence, by 4.03 and $4.04, N(0, p r *)$ is generated by the set of elements $\left[a_{i_{1}}, \cdots, a_{i_{m}}\right]^{p^{k}}$ with $m \geqq 2, k \in\{0, \cdots, \alpha-1\}, k m \neq 2,(k-1) m \delta_{2 r} \neq 2, m+(p-1) k \geqq p r+\delta_{k 0}$ (where $\delta_{u v}=1$ if $u=v$ and 0 if $u \neq v$ ), and $i_{1}>i_{2} \leqq i_{3} \leqq \cdots \leqq i_{m}$, and the elements

$$
\prod_{s=2}^{p r}\left[a_{i_{s}}, a_{i_{1}}, \cdots, a_{i_{s-1}}, a_{i_{s+1}}, \cdots, a_{i_{p r}}\right] \text { with } i_{1} \leqq i_{2} \leqq \cdots \leqq i_{p r} .
$$

An induction on $m$ (using some ideas from the proof of 4.04) then yields the result. 
Observe that an element of $\mathscr{B}$ occurs in at most one of the products in (iii) above. It follows that every element of $N(0, p r *)$ can be uniquely written (up to order) as $\prod_{i=1}^{t} b_{i}^{\beta(i)}$ where the $b_{i}$ are distinct elements of $\mathscr{B}_{p r *}$ and each $\beta(i) \in$ $\{1, \cdots, p-1\}$.

It is a straight-forward consequence of 4.01 and the remarks after the proofs of 4.06 and 4.07 that the $N(\tau, v)$ for $\tau$ in $\{0, \cdots, \alpha-1\}$ and $v$ in $P(p) \backslash\{\omega\}$ are non-trivial and distinct and that the following relations between them hold:

4.08 For all $\tau$,

$$
\begin{aligned}
N(\tau, n) \cap N(\tau+1,1) & = \begin{cases}N(\tau+1, n-p+1) & \text { for } 2 p \leqq n \in P, \\
N(\tau+1, n-p+1) N(\tau+2,1) & \text { for } p<n<2 p, \\
N(\tau+1,2) N(\tau+2,1) & \text { for } 2 \leqq n \leqq p ;\end{cases} \\
N(\tau, p r *) \cap N(\tau+1,1) & = \begin{cases}N(\tau+1, p(r-1)) & \text { for } 1 \neq r \in P, \\
N(\tau+1,2) N(\tau+2,1) & \text { for } r=1 ;\end{cases} \\
\bigcap_{n \in P} N(\tau, n) N(\tau+1,1) & =N(\tau+1,1) .
\end{aligned}
$$

The next step is to prove that every fully invariant subgroup of $H$ can be expressed in terms of the $B(\beta)$ and the $N(\tau, v)$.

4.09 If $V$ is a fully invariant subgroup of $H$, then there is a unique element, call it $\boldsymbol{\beta}(V)$, in $\{0, \cdots, \alpha+1\}$ such that $V=B(\beta(V))(V \cap N(0,1))$.

Observe that if $\mathfrak{Z}$ is a subvariety of $\mathfrak{A}_{p^{\alpha}} \mathfrak{A}_{p}$, then $\boldsymbol{\beta}(\mathfrak{B})$ (see section 2 ) is the same as $\boldsymbol{\beta}(\mathfrak{B}(H))$.

Proof of 4.09. Recall that $N(0,1)$ is the commutator subgroup of $H$. Clearly there is precisely one $\beta$ in $\{0, \cdots, \alpha+1\}$ such that $V N(0,1)=B(\beta) N(0,1)$. Then $a_{1}^{p^{\beta}}=v d$ where $v \in V, d \in N(0,1)$. Applying to this the endomorphism of $H$ which maps $a_{1}$ to $a_{1}$ and all the other generators to the identity yields $a_{1}^{p^{\beta}} \in V$. Thus $B(\beta) \leqq V$ and the result follows.

4.10 For $\tau$ in $\{0, \cdots, \alpha-1\}$, if $V$ is a fully invariant subgroup of $H$ contained in $N(\tau, 1)$, then there is just one element $v(\tau, V)$ of $P(p)$ such that $V=N(\tau, v(\tau, V))(V \cap N(\tau+1,1))$.

Observe that if $\mathfrak{B}$ is a subvariety of $\mathfrak{H}_{p^{x}} \mathfrak{H}_{p}$ then

so that

$$
\boldsymbol{v}(\tau, \mathfrak{B})=\boldsymbol{v}\left(\tau, \mathfrak{B} \vee\left(\mathfrak{N}(\tau, 1) \wedge \mathfrak{A}_{p^{\alpha}} \mathfrak{H}_{p}\right)\right)
$$

$$
\boldsymbol{v}(\tau, \mathfrak{B})=\boldsymbol{v}(\tau, \mathfrak{B}(H) \cap N(\tau, 1)) .
$$

4.10 is proved in two stages. The first will be stated as a separate result. The endomorphism of $H$ which maps $a_{j}$ to $e$ and fixes the other generators will be denoted $\delta_{j}$.

4.11 Let $n-1$ be in $P$ and $\tau$ in $\{0, \cdots, \alpha-1\}$. If $p$ does not divide $n$ or if $p=n=2$, 
there is no fully invariant subgroup of $H$ strictly between $N(\tau, n) N(\tau+1,1)$ and $N(\tau, n-1) N(\tau+1,1)$. Otherwise $n=p r$ and $N(\tau, p r *) N(\tau+1,1)$ is the only fully invariant subgroup of $H$ strictly between them.

Proof. Let $V$ be a fully invariant subgroup of $H$ such that $N(\tau, n) N(\tau+1,1)$ $<V \leqq N(\tau, n-1) N(\tau+1,1)$. There are two cases.

(a) If $V$ contains $w=\prod_{i=1}^{t} b_{i}^{\beta(i)}$ where the $b_{i}$ are distinct elements of $\mathscr{B}$ (of 4.05) of weight $n$, each $\beta(i) \in\left\{p^{\tau}, 2 p^{\tau}, \cdots,(p-1) p^{\tau}\right\}$ and $\operatorname{wt}\left(b_{i}, a_{j}\right)=p$ for some $i$ and some $j$, then $V=N(\tau, n-1) N(\tau+1,1)$.

Clearly it suffices to consider the case $\operatorname{wt}\left(b_{1}, a_{j}\right)=p$. Put $f(0)=0$ and $f(k)=f(k-1)+\mathrm{wt}\left(b_{1}, a_{k}\right)$ and let $\theta$ be the endomorphism of $H$ which maps $a_{k}$ to $a_{f(k-1)+1} \cdots a_{f(k)}$ [to the identity if $f(k-1)=f(k)$ ]. Using 4.02 gives

where

$$
w \theta \prod_{m=1}^{n}\left(l-\delta_{m}\right)=\prod_{s=1}^{p} b(f(j-1)+s, n)^{r} w^{\prime}
$$

$$
r= \pm(p-1) ! \prod_{k \neq j} \mathrm{wt}\left(b_{1}, a_{k}\right) ! \beta(1),
$$

$w^{\prime} \in N(\tau, n) N(\tau+1,1)$ and $b(1, n)$ is interpreted to be the identity. Hence $V$ contains $\prod_{s=1}^{p} b(f(j-1)+s, n)^{p^{\tau}}$ because $p^{\tau+1}$ does not divide $r$. Applying $l-\pi_{1, f(j-1)+2}$ to this and using 4.02 yields that $b(f(j-1)+2, n)^{p^{\tau}}$ is in $V$ and the result follows.

(b) The only products of the form $\prod_{i=1}^{t} b_{i}^{\beta(i)}$ where the $b_{i}$ are distinct elements of $\mathscr{B}$ of weight $n$ and the $\beta(i) \in\left\{p^{\tau}, \cdots,(p-1) p^{\tau}\right\}$ are such that $\operatorname{wt}\left(b_{i}, a_{j}\right)<p$ for all $i, j$.

For $k$ in $P \cup\{0\}$ and $m$ in $\{1, \cdots, p-1\}$ let $I_{k, m}$ be the set of products of the above form in $V$ in which $\mathrm{wt}\left(b_{i}, a_{k}\right) \leqq m$ for all $i$ [take $\mathrm{wt}\left(b_{i}, a_{0}\right)=0$ ], and for all $j$ exceeding $k$ the $\operatorname{wt}\left(b_{i}, a_{j}\right)$ are independent of $i$ and equal to 0 or 1 . Let $V_{k, m}$ be the fully invariant closure in $H$ of $\Pi_{k, m}$ and $N(\tau, n) N(\tau+1,1)$. Clearly $V_{k, p-1} \leqq V_{k+1,1}$ and $V_{k, m} \leqq V_{k, m+1}$ for all $k$ and $m$ in $\{1, \cdots, p-2\}$. If $w \in \Pi_{k+1,1}$, then both $w\left(\imath-\delta_{k+1}\right)$ and $w \delta_{k+1}$ are in $\Pi_{k, p-1}$; hence $w$ is in $V_{k, p-1}$ and $V_{k+1,1}=V_{k, p-1}$. The argument which follows establishes $V_{k, m}=V_{k, m+1}$. Let $\theta, \psi$ be the endomorphisms of $H$ defined by:

$$
\begin{aligned}
& a_{j} \theta= \begin{cases}a_{j} & \text { for } j<k, \\
a_{k} \cdots a_{k+m} & \text { for } j=k, \\
a_{j+m} & \text { for } j>k ;\end{cases} \\
& a_{j} \psi= \begin{cases}a_{j} & \text { for } j<k, \\
a_{k} & \text { for } j \in\{k, \cdots, k+m\}, \\
a_{j-m} & \text { for } j>k+m .\end{cases}
\end{aligned}
$$


It is easy to verify, using 4.02 , that if $w \in \Pi_{k, m+1}$, then $w_{1}=w \theta\left(t-\delta_{k}\right) \cdots$ $\left(l-\delta_{k+m}\right)$ is in $V_{k, 1}$ and $w^{(m+1) !}\left(w_{1} \psi\right)^{-1}$ is in $V_{k, m}$, and hence that $w$ is in $V_{k, m}$. From these equalities it follows that $V=V_{0, p-1}$; that is, $V$ is the fully invariant closure of $N(\tau, n) N(\tau+1,1)$ and the products of the form $\prod_{s=2}^{n} b(s, n)^{\beta(s)}$ (with $\left.\beta(s) \in\left\{0, p^{\tau}, \cdots,(p-1) p^{\tau}\right\}\right)$ which lie in it. If $w=\prod_{s=2}^{n} b(s, n)^{\beta(s)} \in V$, then $w\left(l-\pi_{s, t}\right) \in V$ for all $s, t$ in $\{2, \cdots, n\}$. But $w\left(l-\pi_{s, t}\right)=\left[a_{s}, a_{t}, a_{1}, \cdots\right]^{\beta(s)-\beta(t)}$ by 4.02 , so $V=N(\tau, n-1) N(\tau+1,1)$ or $\beta(s)=\beta(t)$ for all $s, t$ and all relevant $w$. In the latter case $V$ is the fully invariant closure of $N(\tau, n) N(\tau+1,1)$ and $x=$ $\prod_{s=2}^{n} b(s, n)^{p^{\tau}}$. If $n=2$, then $V=N(\tau, n-1) N(\tau+1,1)$. If $n \neq 2$ and $p$ divides $n$, then $V=N(\tau, n *) N(\tau+1,1)$. If $p$ does not divide $n$, then $x\left(l-\pi_{1,2}\right)=b(2, n)^{n p^{\tau}}$ $\in V$ and so $V=N(\tau, n-1) N(\tau+1,1)$.

Proof of 4.10. It follows from 4.06 and 4.07 that if $\mu \neq v$ in $P(p)$, then $N(\tau, v) N(\tau+1,1) \neq N(\tau, \mu) N(\tau+1,1)$. Thus there is at most one $v$ in $P(p)$ such that $V=N(\tau, v)(V \cap N(\tau+1,1))$. If $V \leqq N(\tau+1,1)$, put $v(\tau, V)=\omega$. If $V \leqq N(\tau+1,1)$, then by 4.08 there is an $n$ in $P$ such that $V \leqq N(\tau, n-1) N(\tau+1,1)$ but $V \leqq N(\tau, n) N(\tau+1,1)$, and it follows from 4.11 that $V N(\tau, n) N(\tau+1,1)$ is either (a) $N(\tau, n-1) N(\tau+1,1)$ or (b) $N(\tau, n *) N(\tau+1,1)$.

Case (a): This implies $N(\tau, n-1) \leqq V N(\tau, n) N(\tau+1,1)$. It follows that $N(\tau, m-1) \leqq V N(\tau, m) N(\tau+1,1)$ for all $m$ in $P$ with $m \geqq n$. Hence $N(\tau, n-1) \leqq$ $V N(\tau, p n) N(\tau+1,1)$. Therefore $b(2, n)^{p^{\tau}}=v \prod_{i=1}^{t} b_{i}^{\beta(i)}$ where $v \in V$, the $b_{i}$ are distinct elements of $\mathscr{B}, p^{\tau}$ divides each $\beta(i)$, and for each $i$ either wt $\left(b_{i}\right)>p n$ or $p^{\tau+1}$ divides $\beta(i)$. By a standard argument (applying in turn the mappings $t-\delta_{1}$, $\left.l-\delta_{2}, \cdots\right)$ it can be assumed that, for all $i, \mathrm{wt}\left(b_{i}, a_{j}\right) \geqq 1$ for $j \leqq n$ and $\operatorname{wt}\left(b_{i}, a_{j}\right)$ $=0$ for $j>n$. Hence $\mathrm{wt}\left(b_{i}\right) \geqq n$ and $p^{\tau+1}$ divides $\beta(i)$ for all $i$, because no element $b$ of $\mathscr{B}$ satisfies $\operatorname{wt}(b)>p n$ and $\operatorname{wt}\left(b, a_{j}\right)=0$ for all $j>n$. Thus $b(2, n)^{p^{\tau}} \in V N(\tau+1, n-1)$ and so $N(\tau, n-1) \leqq V N(\tau+1, n-1)$. It follows that $N(\rho, n-1) \leqq V N(\rho+1, n-1)$ for all $\rho \geqq \tau$. Therefore $N(\tau, n-1) \leqq V$. But $V \leqq N(\tau, n-1) N(\tau+1,1)$ and so the result follows with $v(\tau, V)=n-1$.

Case (b): Now $n=p r \geqq 3$ and $N(\tau, p r *) \leqq V N(\tau, p r) N(\tau+1,1)$. By 4.02, $\left[\prod_{s=2}^{p r} b(s, p r), a_{p r+1}\right]\left(l-\pi_{2, p r+1}\right)=\left[a_{2}, a_{p r+1}, a_{1}, \cdots, a_{p r}\right]$. Hence $N(\tau, p r) \leqq$ $V N(\tau, p r+1) N(\tau+1,1)$ and so $N(\tau, p r *) \leqq V N\left(\tau, p^{2} r\right) N(\tau+1,1)$. Therefore arguing as in (a) we obtain that

$$
\prod_{s=2}^{p r} b(s, p r)^{p^{\tau}}=v \prod_{s=2}^{p r} b(s, p r)^{\mu(s)} \prod_{i=1}^{t} b_{i}^{\beta(i)}
$$

where $v \in V$, the $b_{i}$ are elements of $\mathscr{B}$ of weight at least $p r+1$ and $p^{\tau+1}$ divides each $\mu(s)$ and $\beta(i)$. Let $\pi$ denote the automorphism of $H$ which maps $a_{i}$ to $a_{i+1}$ if $2 \leqq i \leqq p r, a_{p r}$ to $a_{2}$, and fixes all other generators. Apply the mapping $\sum_{m=0}^{p r-2} \pi^{m}$ to the last displayed relation above: since $p r-1$ is prime to $p$, it follows that $\prod_{s=2}^{p r} b(s, p r)^{p^{\tau}} \in V N(\tau+1, p r *)$. Hence $N(\tau, p r *) \leqq V N(\tau+1, p r *)$. Then arguing as in (a) shows that the result holds with $v(\tau, V)=p r *$. 
PrOOF OF 2.1. Let $\mathfrak{B}$ be a subvariety of $\mathfrak{A}_{p^{\alpha}} \mathfrak{H}_{p}$. By 4.09 and repeated applications of 4.10 ,

$$
\mathfrak{B}(H)=B(\boldsymbol{\beta}(\mathfrak{B}(H))) \prod_{\tau=0}^{\alpha-1} N(\tau, \boldsymbol{v}(\tau, \mathfrak{B}(H) \cap N(\tau, 1))) .
$$

It follows from the observations after 4.09 and 4.10 that

$$
\mathfrak{B}(H)=B(\boldsymbol{\beta}(\mathfrak{B})) \prod_{\tau=0}^{\alpha-1} N(\tau, \boldsymbol{v}(\tau, \mathfrak{B})) .
$$

Going over to varieties gives the result.

Proof of 2.2. It follows from the argument in the proof of 2.1 that

$$
(\mathfrak{U} \wedge \mathfrak{B})(H)=\mathfrak{U}(H) \mathfrak{B}(H)=B(\beta(\mathfrak{U})) B(\beta(\mathfrak{B})) \prod_{\tau=0}^{\alpha-1} N(\tau, \boldsymbol{v}(\tau, \mathfrak{U})) N(\tau, \boldsymbol{v}(\tau, \mathfrak{B})) .
$$

Since the $B($ )'s and the $N(\tau$,$) 's are linearly ordered,$

$$
(\mathfrak{U} \wedge \mathfrak{B})(H)=B(\min \{\boldsymbol{\beta}(\mathfrak{U}), \boldsymbol{\beta}(\mathfrak{B})\}) \prod_{\tau=1}^{\alpha-1} N(\tau, \min \{\boldsymbol{v}(\tau, \mathfrak{U}), \boldsymbol{v}(\tau, \mathfrak{B})\}) .
$$

It follows from 4.09 that

and

$$
\boldsymbol{\beta}(\mathfrak{U} \wedge \mathfrak{B})=\min \{\boldsymbol{\beta}(\mathfrak{U}), \boldsymbol{\beta}(\mathfrak{V})\}
$$

$$
(\mathfrak{U} \wedge \mathfrak{B})(H) \cap N(0,1)=\prod_{\tau=0}^{\alpha-1} N(\tau, \min \{\boldsymbol{v}(\tau, \mathfrak{U}), \boldsymbol{v}(\tau, \mathfrak{B})\}) .
$$

An induction on $\rho$, using 4.10 , yields

and

$$
\boldsymbol{v}(\rho,(\mathfrak{U} \wedge \mathfrak{B})(H) \cap N(\rho, 1))=\min \{\boldsymbol{v}(\rho, \mathfrak{U}), \boldsymbol{v}(\rho, \mathfrak{B})\}
$$

$$
(\mathfrak{U} \wedge \mathfrak{B})(H) \cap N(\rho+1)=\prod_{\imath=\rho+1}^{\alpha-1} N(\tau, \min \{\boldsymbol{v}(\tau, \mathfrak{U}), \boldsymbol{v}(\tau, \mathfrak{B})\}) .
$$

Hence, by the remark after 4.10 ,

as required.

$$
v(\tau, \mathfrak{U} \wedge \mathfrak{B})=\min \{v(\tau, \mathfrak{U}), v(\tau, \mathfrak{B})\}
$$

Before proving 2.3 we need one more result.

\subsection{For $\beta \geqq 1$}

$$
B(\beta) \cap N(0,1)= \begin{cases}N(\beta-1,1) & \text { for } p=2, \\ N(\beta-1, p *) N(\beta, 1) & \text { for } p \text { odd }\end{cases}
$$

Proof. The result is an easy consequence of the case $\beta=1$ so we only prove that. For $p=2$ this is an immediate consequence of the well-known fact that all 
groups of exponent 2 are abelian. Let $p$ be an odd prime. Since $N(0,1) \geqq B(1) \cap$ $N(0,1) \geqq N(1,1)$, it follows from 4.09 that there is a $v$ in $P(p)$ such that $B(1) \cap$ $N(0,1)=N(0, v) N(1,1)$. There are metabelian groups of exponent $p$ and class precisely $p$ (see [11] Satz 3 or [4] Example 3.2), so $v>p-1$. By 18.4.13 of [6], $\left[a_{2},(p-1) a_{1}\right] \in(B(1) \cap N(0,1)) N(0, p)$. By $4.06,\left[a_{2},(p-1) a_{1}\right] \notin N(0, p) N(1,1)$, so $v<p$. Thus $v=p *$ and the result follows.

ProOF of 2.3. Clearly the set $\Sigma$ is a sublattice of the direct product lattice $\Lambda$. It is a straight-forward matter to calculate using 4.08 and 4.12 that

$$
\boldsymbol{v}\left(\tau, \mathfrak{B}(\beta) \wedge \mathfrak{A}_{p^{\alpha}} \mathfrak{H}_{p}\right)= \begin{cases}\omega\left(\mathfrak{B}(\beta) \wedge \mathfrak{A}_{p^{\alpha}} \mathfrak{A}_{p}\right)=\min \{\beta, \alpha+1\}, \\ p * \text { for } \tau<\beta-1, \\ 1 \quad \text { for } \tau=\beta-1 \text { and } p=2, \\ 1 \quad \text { for } \tau \geqq \beta ;\end{cases}
$$

and $\boldsymbol{\beta}\left(\mathfrak{N}(\tau, v) \wedge \mathfrak{U}_{p^{\alpha}} \mathfrak{A}_{p}\right)=\alpha+1$,

$\boldsymbol{v}\left(\rho, \mathfrak{N}(\tau, v) \wedge \mathfrak{A}_{p^{\alpha}} \mathfrak{A}_{p}\right)= \begin{cases}\omega & \text { for } \rho<\tau, \\ v & \text { for } \rho=\tau, \\ \boldsymbol{v}\left(\rho, \mathfrak{R}(\tau+1, \bar{v}) \wedge \mathfrak{A}_{p^{\alpha}} \mathfrak{A}_{p}\right) & \text { for } \rho>\tau \text { and } v>2 p-1, \\ \bar{v} & \text { for } \rho=\tau+1 \text { and } v \leqq 2 p-1, \\ 1 & \text { for } \rho>\tau+1 \text { and } v \leqq 2 p-1,\end{cases}$

where

$$
\bar{v}= \begin{cases}v & \text { for } v \in\{1, \omega\} \\ v-p+1 & \text { for } v \in P \text { and } v>p, \\ p r & \text { for } v=p(r+1) * \\ 2 & \text { for } 2 \leqq v \leqq p .\end{cases}
$$

Hence $\left(\mathfrak{B}(\beta) \wedge \mathfrak{A}_{p^{\alpha}} \mathfrak{A}_{p}\right) \chi$ and $\left(\mathfrak{N}(\tau, v) \wedge \mathfrak{A}_{p^{\alpha}} \mathfrak{A}_{p}\right) \chi$ belong to $\Sigma$ and so the image of $\chi$ lies in $\Sigma$. Moreover it follows that if $\left(\beta, v_{0}, \cdots, v_{\alpha-1}\right) \in \Sigma$, then

$$
\left(\mathfrak{B}(\beta) \wedge \bigwedge_{\tau=0}^{\alpha-1} \mathfrak{R}\left(\tau, v_{\tau}\right) \wedge \mathfrak{A}_{p^{\star}} \mathfrak{A}_{p}\right) \chi=\left(\beta, v_{0}, \cdots, v_{\alpha-1}\right) .
$$

\section{Proof of Theorems 2 and 5}

ProOf of Theorem 5. Since a group $G$ is nilpotent if it has a nilpotent normal subgroup $N$ such that $G / \mathscr{A}(N)$ is nilpotent (P. Hall [7] Theorem 7), it suffices to prove the theorem for metabelian varieties. Let $\mathfrak{B}$ be a metabelian variety which does not contain $\mathfrak{A}_{p} \mathfrak{A}_{p}$; then there is a positive integer $c$ such that $\mathfrak{J} \wedge \mathfrak{A}_{p} \mathfrak{H}_{p} \subseteq$ $\mathfrak{R}_{c-1}$. We show that every $p$-group in $\mathfrak{B}$ lies in $\mathfrak{R}_{c-1}$. Suppose not; then there 
would be a finitely generated, and therefore finite, $p$-group in $\mathfrak{B} \backslash \mathfrak{R}_{c-1}$. Since all finite $p$-groups are nilpotent, it would follow that there is a $p$-group in $\left(\mathfrak{B} \wedge \mathfrak{R}_{c}\right) \backslash$ $\mathfrak{R}_{c-1}$. The result is therefore a consequence of the following more precise lemma.

LEMMA. If $\mathfrak{B}$ is a metabelian variety such that $\mathfrak{B} \wedge \mathfrak{A}_{p} \mathfrak{H}_{p} \subseteq \mathfrak{R}_{v}$ for some $v$ in $P(p)$, then for each $\mu$ in $P(p) \backslash\{\omega\}$ with $\mu>v$ there is a positive integer $k$ not divisible by p such that $\mathfrak{B} \wedge \mathfrak{R}_{\mu} \subseteq \mathfrak{H}_{k} \mathfrak{R}_{v}$.

Proof. There is nothing to prove if $v=\omega$. If $v \neq \omega$, then it clearly suffices to prove the result when $\mu$ is the first positive integer exceeding $v$-call it $c$. Let $G$ be a free group of $\mathfrak{A} \mathfrak{A} \wedge \mathfrak{R}_{c}$ freely generated by $\left\{g_{1}, \cdots, g_{c}\right\}$, let $V=\mathfrak{B}(G)$ and $K=\mathfrak{R}_{v}(G)$. We will show there is an element $y$ of $K$ such that $y^{p_{w}} \in V$ where $w=\left[g_{1}, \cdots, g_{c}\right]$ if $v=c-1$ and $w=\prod_{s=2}^{c}\left[g_{s}, g_{1}, \cdots, g_{s-1}, g_{s+1}, \cdots, g_{c}\right]$ if $v=c *$. Since $K$ is finitely generated abelian and the fully invariant closure of $w$, it will follow that $K V / V$ is a finitely generated abelian group in which every element has a $p$-th root; hence that $K V / V$ is a finite abelian group of order $k$ not divisible by $p$; and therefore $\mathfrak{B} \wedge \mathfrak{R}_{c} \subseteq \mathfrak{A}_{k} \mathfrak{R}_{v}$ as required. Since $\mathfrak{B} \wedge \mathfrak{A}_{p} \mathfrak{A}_{p} \subseteq \mathfrak{N}_{v}$ it follows that $K \leqq V D$ where $D=\mathfrak{A}_{p} \mathfrak{A}_{p}(G)$ and hence $w=v_{0} d_{0}$ with $v_{0} \in V, d_{0} \in D$. For each $i$ in $\{1, \cdots, c\}$ let $\varepsilon_{i}$ be the endomorphism of $G$ which maps $g_{j}$ to $g_{j}$ for $j \neq i$ and $g_{i}$ to $e$. We now define $v_{1}, \cdots, v_{c} \in V$ and $d_{1}, \cdots, d_{c} \in D$ by $v_{i}=v_{i-1}\left(v_{i-1} \varepsilon_{i}\right)^{-1}$ and $d_{i}=\left(d_{i-1} \varepsilon_{i}\right)^{-1} d_{i-1}$. It is easy to check for all $i$ that $\left(v_{i-1} \varepsilon_{i}\right)\left(d_{i-1} \varepsilon_{i}\right)=e$, $w=v_{i} d_{i}$ and $d_{i} \varepsilon_{j}=e$ for all $j \leqq i$. It follows ([12] 36.32) that $d_{c}$ can be uniquely written in the form $\prod_{s=2}^{c}\left[g_{s}, g_{1}, \cdots, g_{s-1}, g_{s+1}, \cdots, g_{c}\right]^{\beta(s)}$. Let $H$ be the free group of $\mathfrak{A}_{p} \mathfrak{A}_{p}$ defined in section 4. Let $\theta$ be the homomorphism of $G$ into $H / N(0, c)$ defined by $g_{i} \theta=a_{i} N(0, c)$ for all $i$ in $\{1, \cdots, c\}$. Then, as $D \theta=\{N(0, c)\}$,

$$
\prod_{s=2}^{c} b(s, c)^{\beta(s)} N(0, c)=d_{c} \theta=N(0, c),
$$

and so $p$ divides $\beta(s)$ for all $s$. Therefore $d_{c}$ has a $p$-th root $b$ in $\mathfrak{N}_{c-1}(G)$ and $w=$ $v_{c} b^{p}$. If $c=2$ or $p$ does not divide $c$, then $\mathfrak{R}_{c-1}(G)=K$ and the proof is complete. Let $\pi$ denote the automorphism of $G$ which maps $g_{1}$ to $g_{1}, g_{i}$ to $g_{i+1}$ when $1<i<c$, and $g_{c}$ to $g_{2}$; put $\psi=\sum_{m=0}^{c-2} \pi^{m}$. If $p$ divides $c$ and $c \geqq 3$, then applying $\psi$ (cf. the last paragraph of the proof of 4.10) yields $w^{c-1}=v_{c} \psi(b \psi)^{p}$ and $b \psi \in K$. The result follows because $p$ does not divide $c-1$.

ProOf of TheOrem 2. Let $\mathfrak{U}$ be a nilpotent-by-abelian just-non-Cross variety. If $\mathfrak{A} \subseteq \mathfrak{U}$, then $\mathfrak{U}=\mathfrak{A}$. If $\mathfrak{A} \nsubseteq \mathfrak{U}$, then $\mathfrak{U}$ has finite exponent, $t$ say. Hence $\mathfrak{U}$ is generated by its finite groups. We will show that there is a bound, $t^{t}$, on the order of chief factors of finite groups in $\mathfrak{U}$. By the Corollary in [8] applied to the class of finite groups in $\mathfrak{U}$, there is no bound on the class of finite nilpotent groups in $\mathfrak{U}$ and the result follows from Corollary 2 of Theorem 5. Let $H / K$ be a chief factor of a finite group $G$ in $\mathfrak{H}$. Clearly $H / K$ is an elementary abelian $p$-group for some $p$ dividing $t$. Let $C$ be the centralizer of $H / K$ in $G$; then $G / C$ is an abelian group which 
has a faithful irreducible representation over the field of $p$ elements. Hence $G / C$ is cyclic and so has order dividing $t$. Therefore the order of $H / K$ is at most $t^{t}$ as required.

REMARK (added in proof, 9 December, 1970). The problem stated in section 2 has a negative solution on account of the results of Bachmuth, Mochizuki, and Walkup ['A nonsolvable group of exponent 5', Bull. Amer. Math. Soc. 76 (1970), 638-640] and O. Yu. Razmuslov [to appear]: for all primes $p \geqq 5$, there exist nonnilpotent locally finite varieties of exponent $p$. Our Theorem 2 has been superseded by results of J. M. Brady ['On the classification of just-non-Cross varieties of groups', Bull. Austr. Math. Soc. 3 (1970), 293-311; 'On soluble justnon-Cross varieties of groups', ibid. 313-323] and O. Yu. Ol'shanskij [to appear].

\section{References}

[1] G. Birkhoff, Lattice Theory, Third (New) Edition (Amer. Math. Soc., Providence, 1967).

[2] M. S. Brooks, 'On lattices of varieties of metabelian groups', J. Austral. Math. Soc. to appear.

[3] R. A. Bryce, 'Metabelian groups and varieties', Bull. Austral. Math. Soc. 1 (1969), 15-25.

[4] N. D. Gupta and M. F. Newman, 'On metabelian groups', J. Austral. Math. Soc. 6 (1966), $362-368$.

[5] N. D. Gupta and M. F. Newman, 'On metabelian groups', Proc. Internat. Conf. Theory of Groups, Austral. Nat. Univ. Canberra, August 1965, pp. 111-113 (Gordon and Breach, New York, 1967).

[6] M. Hall Jr., The Theory of Groups (Macmillan, New York, 1959).

[7] P. Hall, 'Some sufficient conditions for a group to be nilpotent', Illinois J. Math. 2 (1958), $787-801$.

[8] L. G. Kovács and M. F. Newman, 'Cross varieties of groups', Proc. Roy. Soc. London Ser. A. 292 (1966), 530-536.

[9] L. G. Kovács and M. F. Newman, 'Just-non-Cross varieties', Proc. Internat. Conf. Theory of Groups, Austral. Nat. Univ. Canberra, August 1965, pp. $221-223$ (Gordon and Breach, New York, 1967).

[10] L. G. Kovács and M. F. Newman, 'On varieties of metabelian groups' (in preparation).

[11] H. Meier-Wunderli, 'Metabelsche Gruppen', Comment. Math. Helv. 24 (1951), 1- 10.

[12] Hanna Neumann, Varieties of Groups (Springer, Berlin, 1967).

[13] P. S. Novikov and S. I. Adyan, 'On infinite periodic groups I', Izv. Akad. Nauk S.S.S.R. Ser. Mat. 32 (1968), 212-244.

Australian National University

Canberra, ACT, 2600 